Ergod. Th. \& Dynam. Sys. (1988), 8, 1-15

Printed in Great Britain

\title{
Dynamics induced on the ends of a non-compact manifold
}

\author{
STEVE ALPERN AND V. S. PRASAD \\ Department of Mathematics, London School of Economics, University of London, \\ London WC2A 2AE, England; York University, North York, Canada M3J1P3
}

(Received 26 August 1986)

\begin{abstract}
Let $\mathscr{H}$ denote the group of homeomorphisms of a $\sigma$-compact manifold $M$ which preserve a locally positive non-atomic measure $\mu$. Such a manifold can be compactified by adjoining ideal points called 'ends', collectively denoted by $E$. Every homeomorphism $h$ in $\mathscr{H}$ induces a measure preserving system $\left(E, \mathscr{Q}, \mu^{*}, h^{*}\right)$ where 2 is the algebra of clopen subsets of $E, \mu^{*}$ is a $0-\infty$ measure induced on $E$ by $\mu$, and $h^{*}: E \rightarrow E$ is a $\mu^{*}$-preserving homeomorphism. For any induced homeomorphism $\sigma=f^{*}$, where $f$ belongs to $\mathscr{H}$, define $\mathscr{H}_{\sigma}=\left\{h \in \mathscr{H}: h^{*}=\sigma\right\}$. We prove that ergodicity is generic in $\mathscr{H}_{\sigma}$ for the compact-open topology if and only if $\left(E, \mathscr{2}, \mu^{*}\right.$, $\sigma)$ is incompressible and ergodic. Furthermore $\mathscr{H}_{\sigma}$ contains an ergodic homeomorphism if and only if $\left(E, 2, \mu^{*}, \sigma\right)$ is incompressible. Since the identity on $M$ induces the identity on $E$, which is incompressible, our results establish that every manifold $(M, \mu)$ supports an ergodic $\mu$-preserving homeomorphism.
\end{abstract}

\section{Introduction}

Let $M$ be a metrizable connected $n$-manifold, $n \geq 2$, possibly with boundary. Let $\mu$ be a $\sigma$-finite nonatomic Borel measure on $M$ which is positive on nonempty open sets, finite on compact sets, and zero on the boundary of $M$. We show that in all such cases $(M, \mu)$ can support an ergodic $\mu$-preserving self homeomorphism, although such homeomorphisms need not be typical. As in previous work on this problem, we apply Baire category arguments to various closed subspaces of the topologically complete group $\mathscr{H}=\mathscr{H}(M, \mu)$ of all homeomorphisms of $M$ which preserve $\mu$. We endow $\mathscr{H}$ with the compact-open topology.

In the first result of this type Prasad [P] showed that ergodicity is generic (dense $\left.G_{\delta}\right)$ in $\mathscr{H}(M, \mu)$ when $M$ is Euclidean $n$-space $\mathbb{R}^{n}$ and $\mu$ is Lebesgue measure. Prasad's result parallels the theorem of Oxtoby and Ulam [O-U] which asserts that ergodicity is generic in $\mathscr{H}(M, \mu)$ when $M$ is any compact connected manifold (and $\mu$ is finite) see also [A2], [K-St] and [F]. However, unlike the topological generality of the theorem in the compact case, Prasad's result does not hold for all metrizable (= $\sigma$-compact) manifolds. This was observed by Alpern [A4] who pointed out that the translation $T(\theta, r)=(\theta, r+1)$ of the infinite cylinder $S^{1} \times \mathbb{R}$ could not be approximated by any ergodic homeomorphism, so that ergodicity is not a generic (or even dense) property in $\mathscr{H}\left(S^{1} \times \mathbb{R}\right.$, Lebesgue). To distinguish between the two cases exemplified by $\mathbb{R}^{n}$ and $S^{1} \times \mathbb{R}$ it was necessary in a previous paper [A-P] to consider 
the set $E$ of 'ends' of $M$. In section two we give a formal definition of these ideal points which describe ways of going to infinity on $M$. Euclidean space $\mathbb{R}^{n}, n \geq 2$, has a single end (point at infinity) whereas the cylinder $S^{1} \times \mathbb{R}$ has two ends. We have previously shown that ([A-P, Theorem 3, (iii)]) a necessary and sufficient condition for ergodicity to be generic in $\mathscr{H}$ is that $M$ have at most one end of infinite measure.

To obtain results about manifolds with more than one end we need to exploit the titled 'induced dynamics on ends' in the following sense. Every homeomorphism $h$ in $\mathscr{H}(M, \mu)$ induces a $\mu^{*}$-preserving homeomorphism $h^{*}: E \rightarrow E$, where $\mu^{*}$ is a $0-\infty$ measure on the algebra 2 consisting of clopen subsets of $E$, where $E$ is topologized as described in $\S 2$. The main idea of this paper is to relate the ergodic properties of a homeomorphism $h$ in $\mathscr{H}(M, \mu)$ to the properties of the dynamical system $\left(E, 2, \mu^{*}, h^{*}\right)$ or the topological dynamics of $h^{*}: E \rightarrow E$. In particular we consider the presence or absence, and possible genericity, of ergodicity in the closed subspaces $\mathscr{H}_{\sigma}=\left\{h \in \mathscr{H}(M, \mu): h^{*}=\sigma\right\}$ of $\mathscr{H}(M, \mu)$ where $\sigma=f^{*}$ for some $f$ in $\mathscr{H}$. The following are our main results.

THEOREM 2. $\mathscr{H}_{\sigma}$ contains an ergodic homeomorphism if and only if the induced dynamical system $\left(E, 2, \mu^{*}, \sigma\right)$ is incompressible.

THEOREM 3. Ergodicity is generic in $\mathscr{H}_{\sigma}$ if and only if the induced dynamical system is ergodic and incompressible.

Theorem 2 can be specialized to show that $(M, \mu)$ always supports an ergodic $\mu$-preserving homeomorphism. If $i$ denotes the identity on $M$ then $i^{*}$, the identity on $E$, is obviously incompressible. Hence $\mathscr{H}_{i *}$ contains an ergodic homeomorphism, by Theorem 2 . Thus we have shown the following.

THEOREM 4. $(M, \mu)$ always supports an ergodic $\mu$-preserving homeomorphism.

A new concept introduced in this paper is the 'end-charge' $c_{h}$ induced by any homeomorphism $h$ in $\mathscr{H}(M, \mu)$ on the ends of $M$. Formally, $c_{h}$ is a finitely additive signed measure on the algebra of $h^{*}$-invariant clopen sets of $E$. The intuitive version of the end-charge is the net charge put on each end if $M$ consists of balanced distributions of electrons and protons, each distributed according to the measure $\mu$, of which only the electrons move according to $h$. The details, in a more mathematical version, are in $\S 4$.

In our previous paper [A-P] we considered some of the same problems, but for technical reasons we were forced to restrict our attention to manifolds with only finitely many ends. The present paper is largely independent of that one, although we use the following topological results derived there. We showed [A-P] that any $\sigma$-compact connected manifold is the countable union of an increasing family of compact connected manifolds. This was an application of recent results of Kirby and Siebenmann [K-S] and Quinn [Q]. For this paper we will need the following version.

THEOREM A ([A-P, Lemma 0]). Every compact subset of $M$ is contained in the interior of a set $R$ which has the following properties (which we call a 'separating set'): 
(i) $R$ is a compact connected manifold with boundary, $\operatorname{dim}(R)=\operatorname{dim}(M)$;

(ii) $\mu$ (boundary $R)=0$;

(iii) $M-R$ has no bounded (relatively compact) components.

To avoid confusion we note that the definition of 'separating set' in [A-P] had an additional property with respect to ends, which we do not require here.

\section{The ends $E$ of $M$}

An 'end' of a manifold $M$ is, roughly speaking, a way of going to infinity on $M$. More precisely, an end of $M$ is a function $e$ which assigns to each compact subset $K$ of $M$ a non-empty connected component, denoted $e(K)$, of $M-K$, in such a way that $K_{1} \subset K_{2}$ implies $e\left(K_{2}\right) \subset e\left(K_{1}\right)$. We will denote the set of all ends of $M$ by $E$. For $A \subset E$ we define $A(K)=\bigcup_{e \in A} e(K)$, for any compact set $K$.

The manifold $M$ may be compactified by adjoining the ends $E$ and defining for each compact set $K \subset M$ a typical neighborhood $N_{K}\left(e_{0}\right)$ of an end $e_{0}$ as the set $e_{0}(K) \cup\left\{e \in E: e(K)=e_{0}(K)\right\}[\mathbf{B}-\mathbf{E}]$.

Every compact set $K$ determines an equivalence relation $\stackrel{K}{\sim}$ on $E$ by $e \sim e^{\prime}$ if and only if $e(K)=e^{\prime}(K)$. For each compact $K$ let $\mathscr{P}_{K}$ denote the finite partition of $E$ into equivalence classes modulo $\stackrel{K}{\sim}$ and let $\mathscr{Q}_{K}$ denote the finite algebra generated by $\mathscr{P}_{K}$. (That is, elements of $\mathscr{P}_{K}$ are the atoms of $\mathscr{Q}_{K}$.) Observe that the algebra $\mathscr{Q}=\bigcup_{K} \mathscr{Q}_{K}$ is identical with the family of clopen subsets of $E$, in the relative topology on $E$ of the end-compactification of $M$ described above. Thus $E$ is always totally disconnected.

The measure $\mu$ on $M$ induces a measure on $(E, 2)$ as follows. If $Q$ belongs to 2 then $Q$ belongs to $\mathscr{2}_{K}$ for some separating set $K$. Define $\mu^{*}(Q)=0$ if $\mu(Q(K))<\infty$ and $\mu^{*}(Q)=\infty$ if $\mu(Q(K))=\infty$. This defines a $0-\infty$ measure $\mu^{*}$ on $(E, 2)$. The measure $\mu^{*}$ is nontrivial $\left(\mu^{*}(E) \neq 0\right)$ as long as $\mu(M)=\infty$.

\section{The induced homeomorphism $h^{*}: E \rightarrow E$}

Every homeomorphism $h: M \rightarrow M$ induces a homeomorphism $h^{*}: E \rightarrow E$ such that

$$
\left[h^{*}(e)\right](K)=h\left(e\left(h^{-1}(K)\right)\right)
$$

for all $e \in E$ and compact $K \subset M$. If $h$ preserves $\mu$ (i.e. if $h \in \mathscr{H}$ ) then $h^{*}$ preserves $\mu^{*}$ (though not conversely). Thus every $h \in \mathscr{H}$ induces a measure preserving system $\left(E, 2, \mu^{*}, h^{*}\right)$. To avoid later confusion it should be pointed out here that the measurable sets constitute only an algebra (the algebra 2 of clopen sets of $E$ ), not a $\sigma$-algebra, so the following definitions deserve more than the usual scrutiny.

Definition. The system $\left(E, 2, \mu^{*}, \sigma\right)$ is called compressible if there is a clopen set $Q \in \mathcal{Q}$ with $\mu^{*}(\sigma Q \cap \sim Q)=0$ and $\mu^{*}(Q \cap \sigma(\sim Q))>0$. Otherwise it is called incompressible. The system is called ergodic if for every (invariant) set $I \in \mathscr{Q}$ with $\mu^{*}(I \triangle \sigma I)=0$, either $\mu^{*}(I)=0$ or $\mu^{*}(\sim I)=0$.

As an example, let $E$ denote the totally disconnected space consisting of $-1, \ldots,-1+\frac{1}{3},-1+\frac{1}{2}, 0, \frac{1}{2}, 1-\frac{1}{3}, 1-\frac{1}{4}, \ldots, 1$. Suppose $\sigma$ fixes -1 and +1 and moves 
all other points to the next larger number. Assume $\mu^{*}=\infty$ for all non-empty sets. The reader should check that $\sigma$ is ergodic and compressible.

Proposition 1. Let $h \in \mathscr{H}$ and write $\sigma=h^{*}$. Let $Q_{1}$ and $Q_{2}$ belong to $\mathscr{2}_{K}$ for some separating set $K$. Then $\mu^{*}\left(\sigma Q_{1} \cap Q_{2}\right)=\infty$ if and only if $\mu\left(h\left(Q_{1}(K)\right) \cap Q_{2}(K)\right)=\infty$. Proof. First suppose $\mu^{*}\left(\sigma Q_{1} \cap Q_{2}\right)=\infty$. By the definition of $\mu^{*}$ it follows that in particular $\mu\left(\left(\sigma Q_{1} \cap Q_{2}\right)(B)\right)=\infty$ for $B=h K \cup K$. But

$$
\begin{aligned}
\left(\sigma Q_{1} \cap Q_{2}\right) B & =\left(h^{*} Q_{1}\right) B \cap Q_{2}(B) \\
& =h Q_{1}\left(h^{-1} B\right) \cap Q_{2}(B) \\
& \subseteq h Q_{1} h^{-1}(h K) \cap Q_{2}(K) \quad \text { since } K \subset B \text { and } h K \subset B \\
& =h\left(Q_{1}(K)\right) \cap Q_{2}(K)
\end{aligned}
$$

Therefore $\mu\left(h\left(Q_{1}(K)\right) \cap Q_{2}(K)\right)=\infty$.

Now assume $\mu^{*}\left(\sigma Q_{1} \cap Q_{2}\right)<\infty$, or equivalently $\mu^{*}\left(\left(\sigma Q_{1} \cap Q_{2}\right) R\right)<\infty$ for a separating set $R \supset K \cup h K$. Observe that

$$
\begin{aligned}
h\left(Q_{1}(K)\right) & =h Q_{1} h^{-1}(h K) \\
& =\sigma Q_{1}(h K) \\
& \subset \sigma Q_{1}(R) \cup R,
\end{aligned}
$$

and

Therefore

$$
Q_{2}(K) \subset R \cup Q_{2}(R)
$$

and

$$
h\left(Q_{1}(K)\right) \cap Q_{2}(K) \subset R \cup\left[\left(\sigma Q_{1}\right)(R) \cap Q_{2}(R)\right]
$$

$$
\mu\left(h Q_{1}(K) \cap Q_{2}(K)\right)<\mu(R)+\mu\left(\left(\sigma Q_{1} \cap Q_{2}\right)(R)\right)<\infty .
$$

THEOREM 1. If $\left(E, 2, \mu^{*}, \sigma\right)$ is a compressible system then $\mathscr{H}_{\sigma}$ contains no recurrent (hence no ergodic) homeomorphisms.

Proof. Suppose $h$ belongs to $\mathscr{H}_{\sigma}\left(h^{*}=\sigma\right)$ and some $Q \in \mathscr{Q}$ satisfies $\mu^{*}(\sigma Q \cap \sim Q)=0$ and $\mu^{*}(Q \cap \sigma(\sim Q))>0$. Choose a separating set $K$ so that $Q \in \mathscr{Q}_{K}$ and define $A=h Q(K) \cap \sim(Q(K))$ and $B=h(\overline{Q(K)}) \cap Q(K)$. It follows from Proposition 1 and our assumptions on $Q, h$ and $K$, that $\mu(A)<\infty$ and $\mu(B)=\infty$.

Let $B^{\prime}$ be any subset of $B$ with $\mu\left(B^{\prime}\right)<\infty$ and let $X=A \cup B^{\prime}$. If $h$ is recurrent then it induces a $\mu$-preserving automorphism $\tau: X \rightarrow X$ by $\tau \dot{x}=h^{(m)} x$, where $m \geq 1$ is the least integer with $h^{m} x \in X$. We claim that for all $x$ in $B^{\prime}, \tau x \in A$. To see this suppose on the contrary that $\tau x=h^{m} x \in B^{\prime}$. Then $h^{m} x \in h(\widetilde{Q(K)})$, so $h^{m-1} x \in \widetilde{Q(K)}$. Since $x \in B^{\prime} \subset B \subset Q(K)$ and $h^{m-1} x \in \widehat{Q(K)}$ there is a least $n, 1 \leq n \leq m-1$, for which $h^{n} x \in \widetilde{Q(K)}$. Hence $h^{(n-1)} x \in Q(K)$ and so $\left.h^{n} x \in h(Q(K)) \cap \overline{Q(K}\right)=A$. But then $h^{n} x \in X$ with $n<m$, violating the minimality of $m$. So we have established that $\tau\left(B^{\prime}\right) \subset A$, so that $\mu\left(B^{\prime}\right) \leq \mu(A)$. But since our construction applies to any finite measured subset $B^{\prime}$ of $B$, where $\mu(B)=\infty$, we need only choose $\mu\left(B^{\prime}\right)>\mu(A)$ to force a contradiction. Hence $h$ is not recurrent.

Proposition 2. Let $h \in \mathscr{H}_{\sigma}$ and assume that the system $\left(E, 2, \mu^{*}, \sigma\right)$ is incompressible. For any separating set $K$, let $E_{1}, \ldots, E_{m}$ be an enumeration of the elements of $\mathscr{P}_{K}$ 
having infinite measure. Define an $m \times m$ 0-1 matrix $T=T(h, K)$ by $t_{i j}=1$ if and only if $\mu\left(h\left(E_{i}(K)\right) \cap E_{j}(K)\right)=\infty$. Then $T$ is a recurrent matrix.

Proof. Suppose that for some $i=1, \ldots, m, t_{i i}^{(n)}=0$ for $n=1,2, \ldots$ (Such a state is called transient.) Let $J=\left\{j: t_{i j}^{(p)}=1\right.$ for some $\left.p=1,2, \ldots\right\}$, and define $Q=\bigcup_{j \in J} E_{j}$. By Proposition 1 it follows that $\mu^{*}(\sigma Q \cap \sim Q)=0$ and that $\mu^{*}(Q \cap \sigma(\sim Q)) \geq$ $\mu^{*}\left(Q \cap \sigma E_{i}\right) \geq \mu^{*}\left(E_{i}\right)=\infty$. Thus $\sigma$ is compressible.

\section{The end-charge}

Every $h$ in $\mathscr{H}$ induces on $E$ a charge (finitely additive signed measure) $c=c_{h}$ with respect to the subalgebra $\mathscr{I}=\mathscr{I}_{h^{*}}$ of $h^{*}$-invariant clopen sets. This section is devoted to defining this 'end charge' $c$ and to establishing some of its elementary properties.

For this discussion fix $h$ in $\mathscr{H}$ and let $\sigma$ denote $h^{*}$. Let $\mathscr{I}=\mathscr{I}_{\sigma}$ be the subalgebra of 2 given by $\mathscr{I}=\left\{I \in \mathscr{Q}: \mu^{*}(I \triangle \sigma I)=0\right\}$. For every $I$ in $\mathscr{I}$ there is a separating $K \subset M$ such that $I \in \mathscr{Q}_{K}$. Define

$$
c(I, K)=\mu(h(\widetilde{I(K)}) \cap I(K))-\mu(h(I(K)) \cap \widetilde{I(K})) .
$$

This difference is well defined because both sets have finite measure, which follows from Proposition 1. We now show that the choice of the separating set $K$ is irrelevant.

Proposition 3. Suppose $I$ is a $\sigma=h^{*}$ invariant set of ends belonging to both $\mathscr{Q}_{K}$ and $2_{K^{\prime}}$. Then $c(I, K)=c\left(I, K^{\prime}\right)$, a number which henceforth will be written simply $c(I)$.

Proof. Let $R$ be any separating set containing $K \cup h K \cup h^{-1} K$. Such an $R$ can be found by Theorem A. We will show that $c(I, R)=c(I, K)$. This will establish the Proposition since we can always find an $R$ which has this relationship simultaneously to both $K$ and $K^{\prime}$, so that $c\left(I, K^{\prime}\right)=c(I, R)=c(I, K)$. Let $B=I(K)-I(R)$ and observe that

$$
\begin{aligned}
& h(B)-B=[h(\widetilde{I(R)}) \cap I(R)] \bigcup_{\text {disj }}[\widetilde{I(K)} \cap h(I(K))] \\
& B-h(B)=[h(\widetilde{I(K)}) \cap I(K)] \bigcup_{\text {disj }}[\widetilde{I(R)} \cap h(I(R))] .
\end{aligned}
$$

Therefore $c(I, R)-c(I, K)=\mu(h B-B)-\mu(B-h B)=0$.

Proposition 4. Let $c=c_{h}$ be the end-charge induced by some homeomorphism $h$ in $\mathscr{H}$ on the algebra $\mathscr{I}$ of $h^{*}$-invariant clopen subsets of $E$. Then

(i) $c(E)=0$;

(ii) $\mu^{*}(I)=0 \Rightarrow c(I)=0$;

(iii) if $I_{1}, I_{2} \in \mathscr{I}$ with $I_{1} \cap I_{2}=\varnothing$, then $c\left(I_{1} \cup I_{2}\right)=c\left(I_{1}\right)+c\left(I_{2}\right)$.

Proof. (i) For any separating set $K, E \in \mathscr{Q}_{K}$ and $E(K)=\sim K$. Therefore

$$
c(E)=c(E, K)=\mu[h(K)-K]-\mu[K-h(K)]=0 .
$$

(ii) if $\mu^{*}(I)=0$ then by definition there is a separating set $K$ with $\mu(I(K))<\infty$. Hence

$$
c(I)=c(I, K)=\mu[I(K)-h(I(K))]-\mu[h(I(K))-I(K)]=0 .
$$

(iii) For any $K$ with $I_{1} \in \mathscr{Q}_{K}$ and $I_{2} \in \mathscr{Q}_{K}, I_{1} \cap I_{2}=\varnothing$ implies $I_{1}(K) \cap I_{2}(K)=\varnothing$. For such a $K, c\left(I_{1} \cup I_{2}, K\right)$ clearly equals $c\left(I_{1}, K\right)+c\left(I_{2}, K\right)$. 
We observe that in a trivial sense the finite additivity of $c$ on $\mathscr{I}$ can be extended to countable additivity since whenever $I \in \mathscr{I}$ is the disjoint union of $I_{1}, I_{2}, \ldots$, all but a finite number of those sets must be the empty set.

\section{Topologies on $\mathscr{G}$ and $\mathscr{H}$}

As in [A1] and [A4] our approach is to consider the space $\mathscr{H}=\mathscr{H}(M, \mu)$ as a subspace of the group $\mathscr{G}=\mathscr{G}(M, \mu)$ of all invertible Borel measurable $\mu$-preserving transformations (not necessarily continuous) on $M$. We will use three topologies on $\mathscr{G}$ : the coarse (sometimes called 'weak'), uniform, and compact-open. These are defined below by the following basic neighborhoods, where $g$ and $f$ belong to $\mathscr{G}$, $B$ and $B_{i}$ are finite measured subsets of $M, K$ is compact, $\varepsilon$ and $\lambda$ are positive numbers, $d$ is the metric on $M$, and (finally) $\triangle$ denotes symmetric difference.

Coarse: $\mathscr{V}\left(g, \lambda, B_{1}, \ldots, B_{n}\right)=\left\{f: \mu\left(g B_{i} \triangle f B_{i}\right)<\lambda, i=1, \ldots, n\right\}$;

Uniform: $\mathcal{U}(g, B, \lambda)=\{f: \mu\{x \in B: f(x) \neq g(x)\}<\lambda\}$;

Compact-open: $\mathscr{C}(g, K, \varepsilon)=\{f: d(f(x), g(x))<\varepsilon$ for a.e. $x$ in $K\}$.

The coarse topology is coarser than the other two, and ( $\mathscr{G}$, coarse) and ( $\mathscr{H}$, compact-open) are each topologically complete. Of course the compact-open topology on $\mathscr{G}$ has been defined so that the relative topology on $\mathscr{H}$ is the usual compact-open topology (uniform convergence on compact sets). For this relative topology we may restrict the compact set $K$ to the following type (Proposition 6).

Definition. A separating set $K$ is called h-moving if $\mu(h(I(K)) \cap K)>0$ for every $h^{*}$-invariant clopen set of ends $I$ in $\mathscr{Q}_{K}$.

Proposition 5. Let $\mathscr{H}_{\sigma}=\left\{h \in \mathscr{H}: h^{*}=\sigma\right\}$ and let $\mathscr{I}=\mathscr{I}_{\sigma}$ denote the algebra of $\sigma$ invariant clopen subsets of $E$. For every $h \in \mathscr{H}_{\sigma}$, let $c_{h}$ be the charge on $\mathscr{I}$. For each fixed $I \in \mathscr{I}_{\sigma}$, the map $c_{h}(I): \mathscr{H}_{\sigma} \rightarrow \mathbb{R}$ is continuous in the compact-open topology. Hence the set $\mathscr{H}_{\sigma}^{c}=\left\{h \in \mathscr{H}_{\sigma}: c_{h}=c\right\}$ is closed, for every end-charge $c$ on $\mathscr{H}_{\sigma}$. Furthermore, $\mathscr{H}_{\sigma}^{c}$ is invariant under right composition with any $h \in \mathscr{H}$ of compact support.

Proof. We will prove the stronger assertion that $c(I)$ is in fact continuous in the coarse topology. Fix any compact set $K$ such that $I$ belongs to $\mathscr{I} \cap \mathscr{Q}_{K}$, so that $c(I)=c(I, K)$. If $h_{j} \rightarrow h$ in the coarse topology then

$$
\begin{aligned}
& \mu\left(h_{j}(\widetilde{I(K)}) \cap I(K)\right) \rightarrow \mu(h(\widetilde{I(K)}) \cap I(K)) \\
& \mu\left(h_{j}(I(K)) \cap \widetilde{I(K)}\right) \rightarrow \mu(h(I(K)) \cap \widetilde{I(K)})
\end{aligned}
$$

so that $c_{h_{j}}(I, K) \rightarrow c_{h}(I, K)$ as required.

Next, suppose that $h_{1} \in \mathscr{H}_{\sigma}$ has end-charge $c_{h_{1}}=c$. If $h_{2} \in \mathscr{H}$ has compact support, then $h_{2}(R)=R$ and $h_{2}(I(R))=I(R)$ for some compact $R$ with $I \in \mathscr{Q}_{R}$. Then $c_{h_{1} h_{2}}(I)=$ $c_{h_{1} h_{2}}(I, R)$, and

$$
\begin{aligned}
c_{h_{1} h_{2}}(I, R) & \left.=\mu\left(h_{1} h_{2}(\widetilde{I(R})\right) \cap I(R)\right)-\mu\left(h_{1} h_{2}(I(R)) \cap \widetilde{I(R)}\right) \\
& =\mu\left(h_{1}(\widetilde{I(R)}) \cap I(R)\right)-\mu\left(h_{1}(I(R)) \cap \widetilde{I(R)}\right) \\
& =c_{h_{1}}(I, R)=c_{h_{1}}(I) .
\end{aligned}
$$

Proposition 6. Let $h \in \mathscr{H}, \varepsilon>0$, and a separating set $K$ be given. Then there is an $h^{\prime}$ in $\mathscr{H}$ with compact support such that $\sup _{x \in M} d\left(x, h^{\prime}(x)\right)<\varepsilon$ and $K$ is hh'-moving. 
Consequently there is a sub-basic family of compact-open topology open sets of the form $\mathscr{C}(\mathrm{g}, K, \varepsilon)$ where $K$ is $g$-moving.

Proof. For each $h^{*}$-invariant set of ends $I$ in $\mathscr{I}_{h^{*}} \cap \mathscr{Q}_{K}$ choose distinct topological $n$-balls $B_{I}$ with diameter less than $\varepsilon$ such that $\mu\left(B_{I} \cap I(K)\right)>0$ and $\mu\left(B_{I} \cap \overline{I(\bar{K})}\right)>$ 0 . For each $I$ choose a $\mu$-preserving homeomorphism $h_{I} \in \mathscr{H}$ with support in $B_{I}$ such that $B_{I} \cap I(K)$ is not invariant under $h_{I}$. There are several ways of constructing $h_{I}$. One way is to take $h_{I}$ to be any ergodic $\mu$-preserving homeomorphism of $B_{I}$ which fixes the boundary of $B_{I}$ (extended to $M$ as the identity off $B_{I}$ ). Such an ergodic homeomorphism exists by the results of Oxtoby and Ulam [O-U] mentioned in the introduction. An easier way to construct $h_{I}$ is to use Theorem B [A1, Theorem 5.1] of $\S 6$. Let $h^{\prime}$ be the composition of the $h_{I}$ 's. Then by our construction $h^{\prime}$ is small and $K$ is $h h^{\prime}$-moving.

\section{Approximation by ergodic transformations}

In this section we will prove (Theorem 2 ) that any $\mu$-preserving homeomorphism $h$ of $M$ can be approximated by an ergodic $\mu$-preserving transformation $f$ (not necessarily continuous) in the following sense. Given any $h$-moving compact set $K$, $f$ can be made pointwise close to $h$ on $K$ and set-wise the same as $h$ on all sets $Q(K)$ where $Q \in \mathscr{Q}_{K}$. In the case that $\mu(M)$ is finite (Theorem 1) then the approximation $f$ may be chosen with further properties (such as weak mixing).

For both of the above results we will need the following local approximation result (local meaning finite measure and compact) which was proved earlier [A3, Theorems 2 and 3, and the proof of Theorem 3].

THEOREM B. Let $G$ denote the group of all $\mu$-preserving bijections of a finite Lebesgue space $(X, \Sigma, \mu)$. Let $\tau_{1}, \tau_{2}$ belong to $\mathscr{G}$, with $\tau_{2}$ antiperiodic. Let $\mathscr{A}$ be a finite subalgebra of $\Sigma$ such that the set map $\tau_{1} / \mathscr{A}$ has no non-trivial periodic point (i.e. set). (This means that there is no $A \in \mathscr{A}-\{X, \varnothing\}$ with $\tau_{1}^{i}(A) \in \mathscr{A}$ for $i=1, \ldots, m$, and $\tau_{1}^{m}(A)=A$.) Then there is a conjugate $\tau_{2}^{\prime}=\tau^{-1} \tau_{2} \tau, \tau \in \mathscr{G}$, of $\tau_{2}$ such that $\tau_{2}^{\prime}(A)=\tau_{1}(A)$ for all $A \in \mathscr{A}$. Suppose further that $d$ is a metric on $X$ such that $\mu$ is positive on open sets. Let $Y$ denote the union of all atoms of $\mathscr{A}$ whose $\tau_{1}$ image is relatively compact and connected. Then given any $\delta>0$ we may further ensure that $\tau_{2}^{\prime}$ satisfies $d\left(\tau_{2}^{\prime}(x), \tau_{1}(x)\right)<\delta$ for $\mu$-a.e. $x$ in $Y$.

Proposition 7. Assume $\mu(M)<\infty$. Let $h$ be a $\mu$-preserving homeomorphism of $M$ and let $K$ be any h-moving (compact) subset of $M$. Set $K=M$ if $M$ is compact. Then for any antiperiodic $\mu$-preserving bijection $\theta$ of $M$, and any $\delta>0$, there is a conjugate $f=\tau^{-1} \theta \tau, \tau \in \mathscr{G}(M)$, of $\theta$ which satisfies

(i) $d\left(f^{-1}(y), h^{-1}(y)\right)<\delta$ for $\mu$-a.e. $y$ in $h(K)$;

(ii) $f(K)=h(K)$; and

(iii) $f(Q(K))=h(Q(K))$ for every set of ends $Q \in \mathscr{Q}_{K}$.

Proof. First suppose that $M$ is not compact. Apply Theorem B with $\tau_{1}=h^{-1}, \tau_{2}=\theta^{-1}$ and $\mathscr{A}$ is the algebra consisting of sets of the form $Q(K)$ or $Q(K) \cup K$, where $Q$ varies over $\mathscr{Q}_{K}$. The assumption that $K$ is $h$-moving is what ensures that $\tau_{1} / \mathscr{A}$ has 
no non-trivial periodic point. The automorphism $f \in \mathscr{G}(M)$ defined by $f^{-1}=\tau_{2}^{\prime}$, where $\tau_{2}^{\prime}$ is the conjugate of $\tau_{2}$ given by Theorem $\mathrm{B}$, satisfies the requirements of the proposition. In the case that $K=M$ is compact, the above proof works trivially with $\mathscr{A}=\{K, \varnothing\}$.

Proposition 8. Assume $\mu(M)=\infty$. Let $h$ be a $\mu$-preserving homeomorphism of $M$ which induces an incompressible homeomorphism $\sigma=h^{*}$ on E. Assume that the end-charge $c_{h}$ induced by $h$ on $E$ is identically zero. Then given any $h$-moving subset $K$ of $M$, and any $\delta>0$, there is an ergodic $\mu$-preserving automorphism $f$ (not continuous, in general) of $M$ such that

(i) $d\left(f^{-1}(y), h^{-1}(y)\right)<\delta$ for $\mu$-a.e. $y$ in $h(K)$;

(ii) $f(K)=h(K)$;

(iii) $f(P(K))=h(P(K))$ for all $P \in \mathscr{P}_{K}$.

Proof. Let $\mathscr{P}_{K}=\left\{E_{1}, \ldots, E_{m}, F_{m+1}, \ldots, F_{l}\right\}$ be the partition of $E$ associated with the compact set $K$ (see $\S 2$ ). This means the connected components of $M-K$ are $E_{1}(K), \ldots, E_{m}(K), F_{m+1}(K), \ldots, F_{l}(K)$ where the $E_{i}(K)$ have infinite measure and the $F_{i}(K)$ have finite measure. Proposition 2 says that the $0-1 m \times m$ matrix $T$, defined by $t_{i j}=1$ if and only if $\mu\left(h E_{i}(K) \cap E_{j}(K)\right)=\infty$, is recurrent. Consequently the state space $\{1, \ldots, m\}$ can be partitioned into 'communicating sets' so that the associated submatrices of $T$ are irreducible. Suppose there are $p$ such sets. Then we will relabel the $E_{1}, \ldots, E_{m}$ as $C_{i}^{r}, i=1, \ldots, p, r=1, \ldots, q_{i}$. Let $C_{i}, i=1, \ldots, p$ be the $h^{*}$-invariant sets defined by $C_{i}=\bigcup_{r=1}^{q_{i}} C_{i}^{r}$, and write $\hat{C}_{i}=C_{i}(K), \hat{C}_{i}^{r}=C_{i}^{r}(K)$. Let $\hat{F}=\bigcup_{i=m+1}^{l} F_{i}(K)$. For $i=1, \ldots, p$, let $A_{i}=h \hat{C}_{i} \cap \sim \hat{C}_{i}$ and let $D_{i}=\hat{C}_{i} \cap \sim h \hat{C}_{i}$. The assumption that the end charge induced by $h$ is identically zero implies that for $i=1, \ldots, p, \mu\left(A_{i}\right)=\mu\left(D_{i}\right)$ and the assumption that $K$ is $h$-moving implies that this common number is positive.

Let $X$ be the finite measured (but generally non-compact) set given by

$$
X=K \cup h K \cup \hat{F} \cup h \hat{F} \cup A \cup D,
$$

where $A=\bigcup_{i=1}^{p} A_{i}$ and $D=\bigcup_{i=1}^{p} D_{i}$. Thus $A_{i}$ is the 'arrival from $\hat{C}_{i}$ ' set consisting of all points of $X$ which came from $\hat{C}_{i}$, and similarly $D_{i}$ is the subset of $X$ consisting of points which have 'departed' into $\hat{C}_{i}$. If $y$ is a point of $M$ whose $h$-orbit enters $X$, with $h^{a-1} y \notin X, h^{a} y \in X, h^{a+1} y \in X, \ldots, h^{b} y \in X, h^{b+1} y \notin X$, then $h^{a} y \in A$ and $h^{b} y \in D$. (It is possible, of course, that $a=b$ and $A \cap D \neq \varnothing$.)

The construction of the ergodic approximation $f$ of $h$ is done in two steps. In step 1, using finite measure techniques (particularly Theorem B), we construct an ergodic $\mu$-preserving automorphism $\tilde{f}$ of the finite measure set $X$ satisfying

(i') $d\left(\tilde{f}^{-1}(y), h^{-1}(y)\right)<\delta$ for $\mu$-a.e. $y$ in $h(K)$;

(ii') $\tilde{f}(K)=h(K)$;

(iii') $\tilde{f}\left(F_{i}(K)\right)=h\left(F_{i}(K)\right), i=m+1, \ldots, l$ and $\tilde{f}\left(D_{i}\right)=A_{i} i=1, \ldots, p$.

In step 2 we utilize a skyscraper construction to extend the restriction of $\tilde{f}$ to $X-D$ to the required automorphism $f$ on $M$, using the ergodic transformation $\bar{f}=\tilde{f}_{A}$ induced on $A$ by $\tilde{f}$. Since $f_{A}=\tilde{f}_{A}=\bar{f}$ by construction, it will follow that $f$ is also ergodic. 
Step 1. Define $\tilde{h}: X \rightarrow X$ to be any $\mu$-preserving automorphism of $X$ which agrees with $h$ on $K \cup \bigcup_{i=m+1}^{l} F_{i}(K)$ and maps $D_{i}$ onto $A_{i}$, for $i=1, \ldots, p$. Apply Theorem B to $\tau_{1}=\tilde{h}^{-1}$, with $\tau_{2}$ any ergodic transformation, and $\mathscr{A}$ the finite algebra whose atoms are $h(K), h\left(F_{i}(K)\right), i=m+1, \ldots, l$, and $A_{i}, i=1, \ldots, p$. As in the proof of Proposition 7 the condition that $\tilde{h}^{-1}$ restricted to $\mathscr{A}$ has no nontrivial periodic point (set) follows from the assumption that $K$ is $h$-moving. If we take $\tilde{f}^{-1}: X \rightarrow X$ to be the $\tau_{2}^{\prime}$ (conjugate of $\tau_{2}$ ) given by Theorem $\mathrm{B}$, then $\tilde{f}: X \rightarrow X$ has the required properties listed above as (i') to (iii').

Step 2 . Let $\bar{f}$ be the transformation induced by $\tilde{f}$ on $A$. Since ergodic transformations induce ergodic transformations, $\bar{f}: A \rightarrow A$ is ergodic. The transformation $\tilde{f}: X \rightarrow X$ may be represented as in Figure 1 by a 'skyscraper' construction with $A$ as the base and $D$ as the union of tops, and $\bar{f}$ as the base transformation.

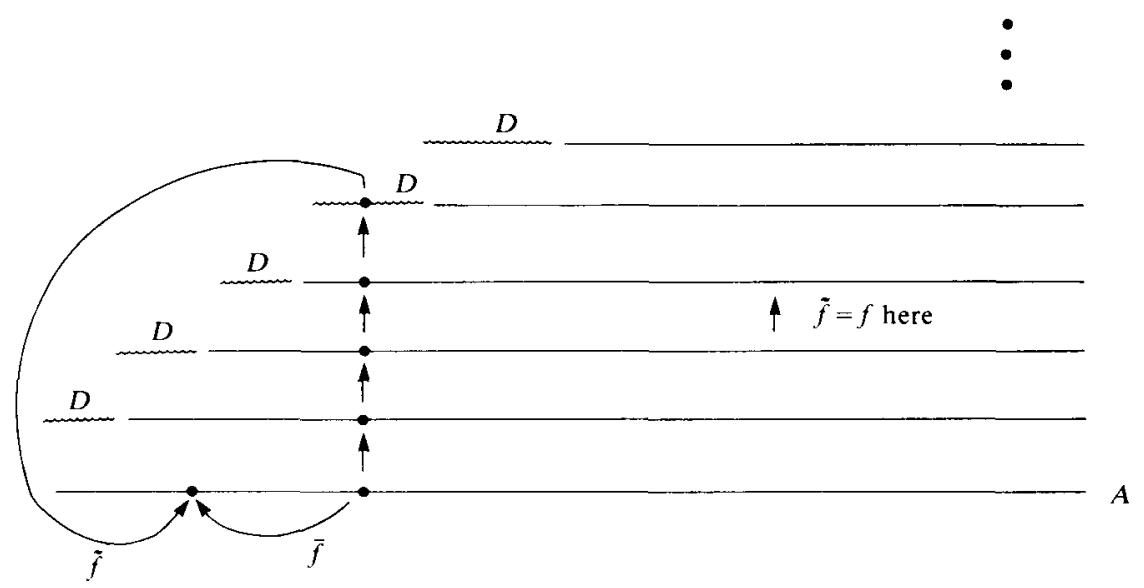

FIGURE 1. Skyscraper built on $A$.

Figure 1 is interpreted as follows. If $x \in X-D$ then $\tilde{f}(x)$ is the point one level up, directly above $x$. If $x \in D$, let $y$ denote the point below $x$ in the base $A$. Then $\tilde{f}(x)=\bar{f}(y)$.

The final stage in the construction of $f$ is to place additional 'levels' above the picture of Figure 1 and fill these levels with all the points of $M-X$. The number of levels above any point of the base $A$ will still be finite, but the sum of the measures of all levels (horizontal sets) will be infinite. The required transformation $f: M \rightarrow M$ is simply the transformation obtained by moving directly up one level, if possible. If $x$ is at the top of the skyscraper, $f$ is defined by $f(x)=\bar{f}(y)$, where $y$ is the point of $A$ directly below $x$. The idea is the same as in the skyscraper version of $\tilde{f}$, except now the picture has infinite measure. Any conservative transformation inducing an ergodic transformation is ergodic, so $f$ is ergodic. Furthermore, any point leaving $X$ by $D_{i}$ will return to $X$ in $A_{i}$, by the construction step 1 . So if we identify all points above $D_{i}$ with points in $\hat{C}_{i}=C_{i}(K)$ the resulting transformation $f$ will satisfy not quite condition (iii) but at least the weaker condition $f\left(C_{i}(K)\right)=h\left(C_{i}(K)\right)$ for $i=1, \ldots, p$. 
To ensure property (iii) we proceed as follows. Fix any $i=1, \ldots, p$ and let $C^{r}$ denote $C_{i}^{r}$, for $r=1, \ldots, n=q_{i}$. Let $B$ denote the $0-1 n \times n$ submatrix of the $m \times m$ matrix $T$ corresponding to this set of $n$ communicating states. As remarked earlier, Proposition 2 guarantees that $B$ is irreducible, where $b_{j k}=1$ iff $\mu\left(h C^{j} \cap C^{k}\right)=\infty$. For the moment we will make the simplifying assumption

$$
b_{j k}=0 \Rightarrow \mu\left(h C^{j} \cap C^{k}\right)=0 .
$$

Since $B$ is irreducible we can find a finite word $\bar{W}=\bar{W}_{1} \bar{W}_{2} \bar{W}_{3} \cdots \bar{W}_{z}$ such that all transitions $\bar{W}_{j} \bar{W}_{j+1}$ are 'legal' $\left(b_{\bar{W}_{j} \bar{W}_{j+1}}=1\right.$ ) and all legal transitions appear in $\bar{W}$ (if $b_{k r}=1$ then for some $j, \bar{W}_{j}=k$ and $\bar{W}_{j+1}=r$ ). Furthermore we can ensure that $\bar{W}$ is cyclic in the sense that $\bar{W}_{z} \bar{W}_{1}$ is a 'legal' transition.

Observe that $A_{i}=h \hat{C}_{i} \cap \sim \hat{C}_{i}$ is the disjoint union of sets $A^{r}=h \hat{C}_{i}^{r} \cap \sim \hat{C}_{i}, r=$ $1, \ldots, n$, and $D_{i}=\hat{C}_{i} \cap \sim h \hat{C}_{i}$ is the disjoint union of sets $D^{s}=\hat{C}_{i}^{s} \cap \sim h \hat{C}_{i}, s=$ $1, \ldots, n$. (Our notation for $A^{r}$ and $D^{s}$ suppresses the dependence on the fixed number $i$, for simplicity). Now fix any column of the skyscraper picture for $\tilde{f}: X \rightarrow X$ with base $A$ (see figure 1). Consider a subcolumn whose top $\Pi$ is a subset of $D^{s}$ and such that $\tilde{f}(\Pi) \subset A^{r}$. See figure 2 .

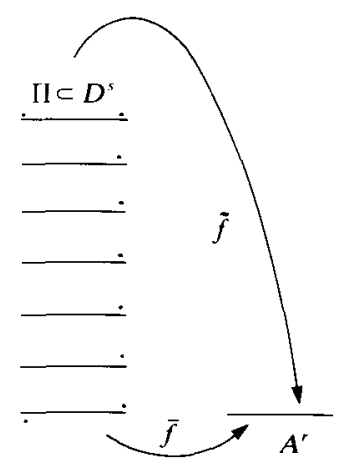

Figure 2

Let $\hat{W}_{0} \hat{W}_{1} \cdots \hat{W}_{\alpha}$ be a word in $\{1, \ldots, n\}$ with legal $B$-transitions and $\hat{W}_{0}=s$, $\hat{W}_{\alpha}=\bar{W}_{z}, \alpha \leq n$. Let $\check{W}_{0} \check{W}_{1} \check{W}_{2} \ldots \check{W}_{\beta}$ be another word in $\{1, \ldots, n\}$ with legal $B$-transitions and $\check{W}_{0}=\bar{W}_{z}, \check{W}_{\beta}=r, \beta \leq n$. Then the word

$$
W^{*}=\hat{W}_{1} \hat{W}_{2} \cdots \hat{W}_{\alpha} \bar{W} \bar{W} \cdots \bar{W} \check{W}_{1} \check{W}_{2} \cdots \check{W}_{\beta}
$$

has all legal $B$ transitions regardless of how many repetitions of the word $\bar{W}$ it contains. So we add column levels above $\Pi$ (of width $\mu(\Pi)$ ) and label each with an integer in $\{1, \ldots, n\}$ according to the word $W^{*}$. We repeat this process above different column tops of type $\Pi$, choosing the number of repetitions of $\bar{W}$ so as to guarantee that the total measure of the added column levels is infinite. This means in particular that the measure of the added column levels labeled by the word $\bar{W}$ is infinite, since the measure of the remaining levels is bounded by $2 n \mu\left(D_{i}\right)$. If $b_{r s}=1$ the set of added column levels whose label is $s$ and which lie above a column level labeled $r$ has infinite measure and may be identified, in a $\mu$-preserving manner, with the set $\hat{C}_{i}^{s} \cap h \hat{C}_{i}^{r}=C_{i}^{s}(K) \cap h C_{i}^{r}(K)$. Hence it follows that $f\left(C_{i}^{r}(K)\right)=$ 
$h\left(C_{i}^{r}(K)\right)$ for $r=1, \ldots, n$, so that condition (iii) is satisfied, completing the proof based on the additional assumption $(*)$.

We now show how to amend the construction if $(*)$ does not hold. Suppose $\gamma=\mu\left(h C^{j} \cap C^{k}\right)>0$ for some $j, k$ with $b_{j k}=0$. We need some column levels labeled $k$ which lie above column levels labeled $j$. Let $\tilde{W}$ be any word in $\{1, \ldots, n\}$ which begins with $\bar{W}_{1}$, ends with $\bar{W}_{z}$, and has all legal $B$-transitions except for one $j$ followed by a $k$. Suppose $\tilde{W}$ has length $L$. Let $\Pi$ be some column top, as in our previous construction, with width ( $\mu$-measure) $\theta$. Choose $\rho<\theta$ and an integer $N$ with $\rho N=\gamma=\mu\left(h C^{j} \cap C^{k}\right)$. Between a column level above $\Pi$ labeled $\bar{W}_{z}$ and the next higher one labeled $\bar{W}_{1}$, add $N L$ column levels of width $\rho$, labeled as $\tilde{W} \tilde{W} \ldots \tilde{W}$ ( $N$ repetitions). Then the column levels labeled $k$ which are above levels labeled $j$ have measure $\gamma$ and can be identified with $h C^{j} \cap C^{k}$. This completes the proof without assuming $(*)$. See figure 3 .

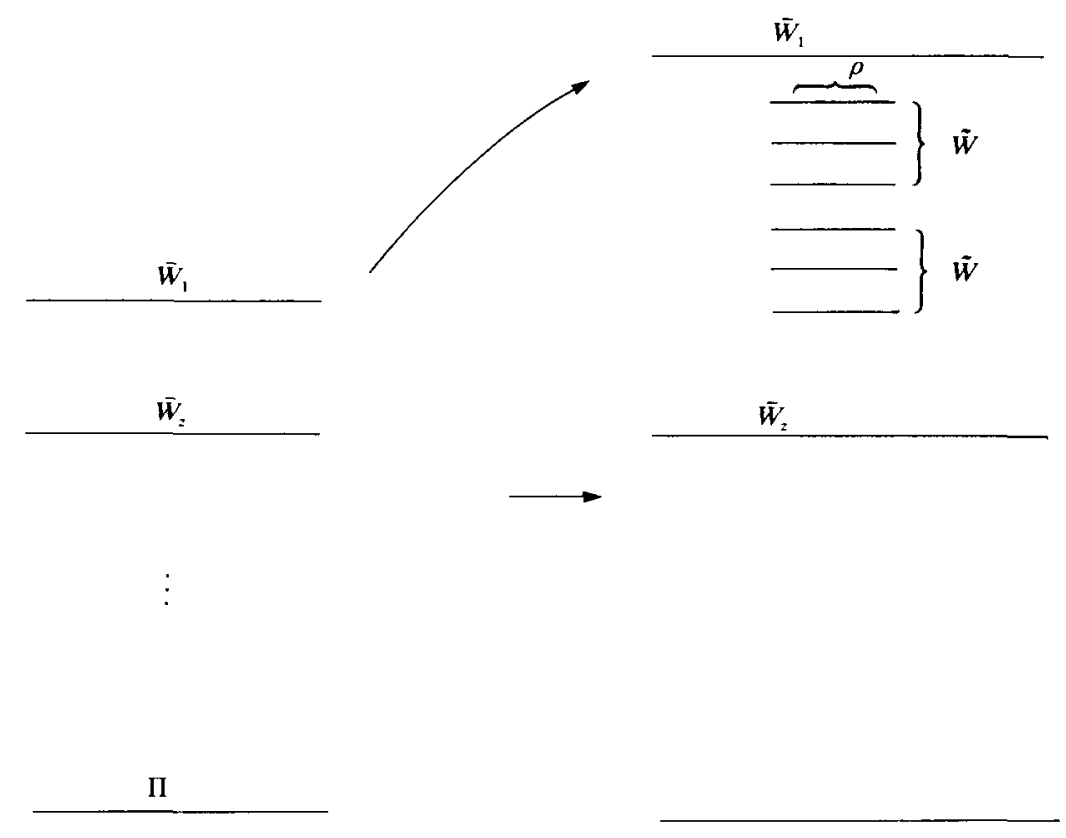

Figure 3

\section{Approximation of measurable by continuous transformations}

In the previous section we showed (Propositions 7 and 8 ) that any $\mu$-preserving homeomorphism of $M$ could be approximated, uniformly on a compact set $K$ and setwise on the open sets $Q(K)$, by an ergodic $\mu$-preserving transformation $f$ of $M$. In this section we deal with the problem that $f$ is not a homeomorphism, $f \in$ $\mathscr{G}(M)-\mathscr{H}(M)$. We will need to show that the non-homeomorphism $g=h^{-1} f$ can be approximated in the coarse topology by a homeomorphism $h^{\prime}$ which, like $h^{-1} f$, is small on $K$. Then $h h^{\prime}$ will be near $h$ on the set $K$, hence close to $h$ in the compact-open topology on $\mathscr{H}(M)$. Also $h h^{\prime}$ will be near $f$ in the coarse topology, hence it will be 'nearly ergodic'. The required result is Theorem 3.2 of [A1] which 
says that any $\mu$-preserving bijection of $K$ (compact set, finite measure) which moves all points a distance less than $\delta$ can be approximated in the coarse topology by a $\mu$-preserving homeomorphism which moves all points a distance less than $\delta$. However for technical reasons we prefer to use the stronger result below (Theorem C) which is obtained by combining Theorem 3.2 of [A1] with the "measure-preserving Luzin Theorem' of J. C. Oxtoby [O] and H. E. White, Jr. [W] which asserts that $\mathscr{H}(K)$ is dense in $\mathscr{G}(K)$ in the uniform topology (as defined in $\S 5$ ).

THEOREM C (Theorem 5.1 of $[\mathbf{A 1}]$ ). Let $(R, d)$ be a compact connected n-manifold with the property that if $d(x, y)<\varepsilon$ there is a connected open set containing $x$ and $y$ with diameter less than $\varepsilon$. Let $\mu$ be a finite Borel measure on $R$ which is non-atomic, locally positive, and zero for the boundary of $R$. Then given any $\mu$-preserving bijection $g: R \rightarrow R$ with ess $\sup _{x \in R} d(x, g(x))<\varepsilon$ and any $\lambda>0$, there is a $\mu$-preserving homeomorphism $h: R \rightarrow R$ which fixes the boundary of $R$ and satisfies $\sup _{x \in R} d(x, g(x))<\varepsilon$ and $\mu\{x: h(x) \neq g(x)\}<\lambda$.

Proposition 9. Let $K$ be a separating subset of $M$ and let $g: M \rightarrow M$ be a $\mu$-preserving bijection satisfying

(i) $g(K)=K$;

(ii) $d(x, g(x))<\delta$ for $\mu$-a.e. $x$ in $K$;

(iii) $g(P(K))=P(K)$ for all $P \in \mathscr{P}_{K}$.

Then any uniform (and hence any coarse) topology neighborhood $\mathcal{U}$ of $g$ contains $a$ homeomorphism $h \in \mathscr{H}$ with compact support satisfying (i)-(iii) with $h$ replacing $g$.

Proof. Let $\mathscr{U}(g, B, \lambda)=\{f \in \mathscr{G}: \mu\{x \in B: f(x) \neq g(x)\}<\lambda\}$ be a uniform topology neighborhood of $g$, where $\mu(B)<\infty$ and $\lambda>0$. For every $P \in \mathscr{P}_{K}$ define $B_{P}=B \cap$ $P(K)$ and choose $\lambda_{P}>0$ so that $\sum_{P \in P_{K}} \lambda_{P}<\lambda$. For each $P \in \mathscr{P}_{K}$ choose $B_{P}^{\prime} \subset B_{P}$ so that $\mu\left(B_{P}-B_{P}^{\prime}\right)<\lambda_{P} / 2$ and $B_{P}^{\prime} \cup g B_{P}^{\prime}$ is a relatively compact subset of $P(K)$. It is hypothesis (iii) that makes this possible. By Theorem A there is a compact connected $n$-manifold $(\operatorname{dim} M=n) K_{P} \subset P(K)$ with boundary measure zero which contains $B_{P}^{\prime} \cup g B_{P}^{\prime}$ in its interior. Let $g_{P}$ be any $\mu$-preserving automorphism of $K_{P}$ onto itself which agrees with $g$ on $B_{P}^{\prime}$. Now apply Theorem $C$ to the restriction of $g$ to $K$, with distance bound $\delta$ and measure error $\lambda / 4$. This yields a $\mu$-preserving, boundary fixing homeomorphism $h_{K}: K \rightarrow K$ satisfying conditions (i) and (ii) and $\mu\{x \in$ $\left.K: h_{K}(x) \neq g(x)\right\}<\lambda / 4$. Similarly we apply Theorem $C$ to each map $g_{P}: K_{P} \rightarrow K_{P}$, but without any distance bound in either the hypothesis or conclusion. This yields $\mu$-preserving homeomorphisms $h_{P}: K_{P} \rightarrow K_{P}$ which fix the boundary of $K_{P}$ and satisfy $\mu\left\{x \in K_{P}: h_{P}(x) \neq g_{P}(x)\right\}<\lambda_{P} / 4$. Let $h \in \mathscr{H}$ be the $\mu$-preserving homeomorphism which agrees with $h_{K}$ on $K$ and $h_{P}$ on $K_{P}, P \in \mathscr{P}_{K}$, and is the identity elsewhere.

\section{Category theorems}

In this section we prove the category theroms stated in the introduction.

Proposition 10. If $\left(E, 2, \mu^{*}, \sigma\right)$ is incompressible then the ergodic homeomorphisms form a dense $\mathscr{G}_{\delta}$ subset of the set $\mathscr{H}_{\sigma}^{0}$ consisting of all $h \in \mathscr{H}$ with $h^{*}=\sigma$ and $c_{h} \equiv 0$, with respect to the compact-open topology. 
Proof. This statement is vacuous if $\mathscr{H}_{\sigma}$ is empty, so we exclude this possibility. We may assume $\mu(M)=\infty$, since Proposition 9 proves a stronger result when $\mu(M)<\infty$.

Choksi and Kakutani [C-K] have shown that the subset $\mathscr{E}$ of $\mathscr{G}=\mathscr{G}(M, \mu)$ consisting of ergodic automorphisms is a $\mathscr{G}_{\delta}$ subset in the coarse topology. So we may write $\mathscr{E}=\bigcap_{i=1}^{\infty} \mathscr{V}_{i}$ where each $\mathscr{V}_{i}$ is a coarse topology open subset of $\mathscr{G}$ which contains $\mathscr{E}$. Since the compact-open topology is finer than the coarse topology, it follows that each set $\mathscr{V}_{i} \cap \mathscr{H}_{\sigma}$ is open in the relative compact-open topology on $\mathscr{H}_{\sigma}$. Since the end-charge is continuous on $\mathscr{H}_{\sigma}$ (Proposition 5) it follows that $\mathscr{H}_{\sigma}^{\circ}$ is a closed subset of the topologically complete space $\mathscr{H}$ (compact-open topology). Hence the proposition will be a conseqence of the Baire Category Theorem if we can show that each set $\mathscr{V}_{i} \cap \mathscr{H}_{\sigma}^{0}$ is a compact-open topology dense subset of $\mathscr{H}_{\sigma}^{0}$.

So we must show that $\mathscr{V} \cap \mathscr{H}_{\sigma}^{0} \cap \mathscr{C} \neq \varnothing$ for any compact-open neighborhood $\mathscr{C}=\mathscr{C}(h, K, \varepsilon)$ (see section 5) with $h \in \mathscr{H}_{\sigma}^{0}$ and any coarse topology open set $\mathscr{V}$ containing $\mathscr{E}$ (in particular, $\mathscr{V}=\mathscr{V}_{i}$ ). By Theorem A and Proposition 6 we may assume that $K$ is an $h$-moving separating set. Let $\delta=\omega(\varepsilon)$ where $\omega$ denotes the uniform modulus of continuity of $h$ on $K$. Let $f \in \mathscr{G}(M)$ be the ergodic automorphism of $M$ given by Proposition 8, which approximates $h$ as follows:

(i) $d\left(f^{-1}(y), h^{-1}(y)\right)<\delta$ for $\mu$-a.e. $y$ in $h(K)$;

(ii) $f(K)=h(K)$;

(iii) $f(P(K))=h(P(K))$ for all $P \in \mathscr{P}_{K}$.

Set $g=h^{-1} f$. Since $f \in \mathscr{E} \subset \mathscr{V}$ it follows that $g$ belongs to the coarse topology open set $h^{-1} \mathscr{V}$. Furthermore $g(K)=K$ and $g(P(K))=P(K)$ for all $P \in \mathscr{P}_{K}$. For all $x$ in $K$,

$$
d(x, g(x))=d\left(x, h^{-1} f(x)\right)=d\left(f^{-1} f(x), h^{-1} f(x)\right)<\delta
$$

by property (i) above. Therefore we may apply Proposition 9 which approximates $g=h^{-1} f$ by a $\mu$-preserving homeomorphism $h^{\prime}: M \rightarrow M$ with compact support which belongs to the coarse topology open set $h^{-1} \mathscr{V}$ and satisfies $d\left(x, h^{\prime}(x)\right)<\delta$ for all $x$ in $K$. The homeomorphism $h h^{\prime}$ is obviously in $\mathscr{V}$, since $h^{\prime}$ is in $h^{-1} \mathscr{V}$. Also $h h^{\prime}$ belongs to $\mathscr{C}=\mathscr{C}(h, K, \varepsilon)$ by choice of $\delta=\omega(\varepsilon)$. Finally, by Proposition $5, h h^{\prime}$ belongs to $\mathscr{H}_{\sigma}^{0}$ because $h^{\prime}$ has compact support. Thus we have established that $\mathscr{V} \cap \mathscr{H}_{\sigma}^{0} \cap \mathscr{C}$ is not empty, as it contains $h h^{\prime}$.

Proposition 11. Assume $\mu(M)<\infty$. Then for any conjugate-invariant property which defines a dense $\mathscr{G}_{\delta}$ subset $\mathscr{F}$ of $\mathscr{G}$ with respect to the coarse topology, $\mathscr{F} \cap \mathscr{H}$ is a dense $\mathscr{G}_{\delta}$ subset of $\mathscr{H}=\mathscr{H}(M)$ with respect to the compact-open topology.

Proof. Since $\mathscr{F}$ and $\mathscr{E}$ are dense $\mathscr{G}_{\delta}$ subsets of $\mathscr{G}$ in the coarse topology, so is $\mathscr{F} \cap \mathscr{E}$. Hence $\mathscr{F}$ contains an ergodic automorphism $\theta \in \mathscr{G}$, and consequently the whole conjugacy class of $\theta$. The rest of the proof now is the same as the proof of Proposition 9, except that Proposition 7 is invoked instead of Proposition 8.

Lemma 1. Suppose $K$ and $R$ are separating sets of $M$ with $K$ in the interior of $R$. Suppose $e$ and $e^{\prime}$ are ends of infinite measure with $e \stackrel{K}{\sim} e^{\prime}$ but $e^{R} e^{\prime}$. Then for any $\alpha \in \mathbb{R}$ there is a homeomorphism $\hat{h}=\hat{h}\left(e, e^{\prime}, \alpha\right)$ which is the identity on $K$, is endpreserving $\left(\hat{h}^{*}=i d e n t i t y\right)$ and satisfies $c_{\hat{h}}(Q)=\alpha$ for any $Q \in \mathscr{Q}_{R}$ with $e^{\prime} \in Q$ and $e \not Q$. 
Proof. A tube $\mathbb{R} \times[0,1]^{n-1}(n=\operatorname{dim} M)$ is embedded in $M-K$ which goes from $e$ (at $-\infty$ ) to $e^{\prime}($ at $\infty)$. This can be done so that Lebesgue measure goes into $\mu$ (see [B-E]). The homeomorphism $\hat{h}$ moves points to the 'right' in the tube tapering off to the identity on the boundary of the tube, and the identity off the tube. Any required 'net flow to the right' of $\alpha$ can clearly be achieved.

Proposition 12. Let $\sigma=h^{*}$ for some $h \in \mathscr{H}$, and suppose $\left(E, 2, \mu^{*}, \sigma\right)$ is incompressible. Then there is a homeomorphism $\bar{h} \in \mathscr{H}_{\sigma}$ such that the induced end-charge $c_{\bar{h}}$ is identically zero.

Proof. By Theorem A we may write $M=\bigcup_{i=1}^{\infty} K_{i}$ where the $K_{i}$ are separating sets with $K_{i}$ in the interior of $K_{i+1}$.

Using a finite composition of the 'flows' of Lemma 1 we may easily obtain an end-preserving homeomorphism $\hat{h}_{1}$ such that the end-charge of $\hat{h}_{1} h$ is identically zero on all $\sigma$-invariant subsets of $\mathscr{2}_{K_{1}}$. Then we can find an end-preserving homeomorphism $\hat{h}_{2} \in \mathscr{H}$ which is the identity on $K_{1}$ such that $\hat{h}_{2} \hat{h}_{1} h$ induces an end-charge which is 0 on all $\sigma$-invariant subsets of $\mathscr{2}_{K_{2}}$. The limit of this process is the required homeomorphism $\bar{h}$.

THEOREM 2. Let $\left(E, \mathscr{Q}, \mu^{*}, \sigma\right)$ be the end dynamical system induced by some $h \in \mathscr{H}$, i.e. $h^{*}=\sigma$. Let $\mathscr{H}_{\sigma}=\left\{g \in \mathscr{H}: g^{*}=\sigma\right\}$. Then $\mathscr{H}_{\sigma}$ contains an ergodic homeomorphism if and only if $\left(E, \mathscr{2}, \mu^{*}, \sigma\right)$ is incompressible.

Proof. The 'only if' part was already established in Theorem 1 by showing that if $\sigma$ is compressible then $\mathscr{H}_{\sigma}$ contains no recurrent homeomorphism. Conversely, Proposition 12 shows that $\mathscr{H}_{\sigma}^{0}$ is not empty, so that Proposition 10 guarantees an ergodic homeomorphism. If $\mu(M)<\infty$ then $\sigma$ is always incompressible, and ergodic homeomorphisms are dense in $\mathscr{H}_{\sigma}$.

Theorem 3. Suppose $\sigma=h^{*}$ for some $h \in \mathscr{H}$. Then ergodicity is generic (dense $\mathscr{G}_{\delta}$ ) in $\mathscr{H}_{\sigma}$ if and only if $\left(E, 2, \mu^{*}, \sigma\right)$ is ergodic and incompressible.

Proof. If $\sigma$ is ergodic and incompressible then the end charge of any $g \in \mathscr{H}_{\sigma}$ is identically zero by Proposition 4(ii). Thus $\mathscr{H}_{\sigma}^{0}=\mathscr{H}_{\sigma}$ and the result follows from Proposition 10. If $\sigma$ is not incompressible the result follows from Theorem 1. If $\sigma$ is incompressible but not ergodic then for some separating set $K$, we can find a $\sigma$-invariant $I \in \mathscr{Q}_{K}$ with $\mu^{*}(I) \neq 0$ and $\mu^{*}(\sim I) \neq 0$. Consequently we may choose infinite measure ends $e \in I$ and $e^{\prime} \in \sim I$ and construct $\hat{h}=\hat{h}\left(e, e^{\prime}, 1\right)$ using Lemma 1. If $h^{\prime}$ is any homeomorphism in $\mathscr{H}_{\sigma}$ which is in some compact-open neighborhood $\mathscr{C}$ of $\hat{h}$, then the induced end charge $c^{\prime}=c_{h}$, will satisfy $c^{\prime}(I)>\frac{1}{2}$. The same proof used in Theorem 1 now shows that $h^{\prime}$ cannot be recurrent. Hence the compact-open neighborhood $\mathscr{C}$ contains no ergodic homeomorphisms, so ergodicity is not generic.

CoRollary 1. Ergodicity is generic in $\mathscr{H}_{\sigma}$ if the restriction of $\sigma$ to the set $E_{\infty}$, consisting of ends of infinite measure, is transitive. (An end $e \in E$ belongs to $E_{\infty}$ iff $\mu(e(K))=\infty$ for every compact set $K \subset M$.)

Proof. For such a $\sigma$, the system $\left(E, 2, \mu^{*}, \sigma\right)$ is ergodic and incompressible. 
Corollary 2. If $\sigma$ is transitive on $E$ then ergodicity is generic in $\mathscr{H}_{\sigma}$.

Proof. This is a special case of Corollary 1, when all ends have infinite measure.

\section{REFERENCES}

[A1] S. Alpern. Approximation to and by measure preserving homeomorphisms. J. London Math. Soc. 18 (1978), 305-315.

[A2] S. Alpern. Generic properties of measure preserving homeomorphisms. Ergodic Theory, Proceedings Oberwolfach 1978, Springer Lecture Notes in Math. 729 (1979), 16-27.

[A3] S. Alpern. Return times and conjugates of an antiperiodic transformation. Ergod. Th. \& Dynam. Sys. 1 (1981), 135-143.

[A4] S. Alpern. Nonstable ergodic homeomorphisms of $\mathbb{R}^{4}$. Indiana U. Math. J. 32 (1983), 187-191.

[A-P] S. Alpern \& V. S. Prasad. End behaviour and ergodicity for homeomorphisms of manifolds with finitely many ends. Canadian J. Math. 39 (1987), 473-492.

[B-E] R. Berlanga \& D. Epstein. Measures on sigma compact manifolds and their equivalences under homeomorphisms. J. London Math. Soc. 27 (1983), 63-74.

[C-K] J. Choksi \& S. Kakutani. Residuality of ergodic measurable transformations and of ergodic transformations which preserve an infinite measure. Indiana U. Math. J. 28 (1979), 453-459.

[F] A. Fathi. The structure of homeomorphisms preserving a good measure. Ann. Scient. Ec. Norm. Sup. 13 (1980), 45-93.

[K-St] A. Katok \& A. Stepin. Metric properties of measure preserving homeomorphisms. Uspekhi Math. Nauk 25 (1970), 193-222; translated in Russian Math. Surveys 25 (1970), 191-220.

[K-S] R. Kirby \& L. Siebenmann. Foundational Essays on Topological Manifolds, Smoothings and Triangulations. Annals of Math. Studies No. 88, Princeton Univ. Press, 1977.

[O] J. Oxtoby. Approximation by measure preserving homeomorphisms. Recent Advances in Topological Dynamics, Springer Lecture Notes in Math. 318 (1973), 206-217.

[O-U] J. Oxtoby \& S. Ulam. Measure preserving homeomorphisms and metrical transitivity. Ann. of Math. 42 (1941), 874-920.

[P] V. S. Prasad. Ergodic measure preserving homeomorphisms of $\mathbb{R}^{n}$, Indiana U. Math. J. 28 (1979), 859-867.

[Q] F. Quinn. Ends of maps, III. J. Diff. Geom. 17 (1982) 503-521.

[W] H. E. White, Jr. The approximation of one-one measurable transformations by measure preserving homeomorphisms. Proc. Amer. Math. Soc. 44 (1974), 391-394. 